\title{
COLANGIOPANCREATOGRAFIA ENDOSCÓPICA RETRÓGRADA INTRAOPERATÓRIA COMO ALTERNATIVA NO TRATAMENTO DE COLEDOCOLITÍASE
}

\section{Intraoperative endoscopic retrograde cholangiopancreatography as an alternative for choledocholithiasis treatment}

\author{
Eric ETTINGER Jr² , Paulo Cezar Galvão do AMARAL ${ }^{2}$, Euler ÁZARO-FILHO, \\ Marcos Fraga FORTES ${ }^{2}$, Heron Crusoé CANGUSSU ${ }^{2}$, Edvaldo FAHEL ${ }^{1}$
}

ABCDDV/528

Ettinger Jr E, Amaral PCG, Azaro-Filho E, Fortes MF, Cangussu HC, Fahel E. Colangiopancreatografia endoscópica retrógrada intraoperatória como alternativa no tratamento de coledocolitíase ABCD Arq Bras Cir Dig 2007;20(1):65-7.

RESUMO - Introdução - O tratamento da coledocolitíase continua controverso, podendo ser realizado por pancreatocolangiografia retrógrada endoscópica pré-operatória seguida de videolaparocolecistectomia ou por videolaparocolecistectomia com exploração de vias biliares. Há relato na literatura de taxa de insucesso da pancreatocolangiografia retrógrada endoscópica pré-operatória em torno de $6 \%$. Relato do caso - Paciente de 45 anos, admitida na emergência com quadro de dor abdominal em epigástrio e hipocôndrio direito. A ultra-sonografia da admissão evidenciou coledocolitíase, sendo encaminhada para pancreatocolangiografia retrógrada endoscópica pré-operatória onde não houve sucesso na cateterização da papila. Optou-se então pela realização de videolaparocolecistectomia com exploração de via biliar por pancreatocolangiografia retrógrada endoscópica pré-operatória intra-operatoria com a cateterização da papila direcionada por fio guia passado pelo ducto cístico. Foi realizada duodenoscopia com captura do fio guia que serviu para a passagem do papilótomo dando seqüência à pancreatocolangiografia retrógrada endoscópica pré-operatória e retirada dos cálculos. A paciente evoluiu satisfatoriamente tendo alta no $2^{\circ}$ dia do pós-operatório. Conclusão - A abordagem da coledocolitíase por pancreatocolangiografia retrógrada endoscópica intra-operatória seguida de videolaparocolecistectomia, mostrou-se segura e eficaz.

DESCRITORES - Coledocolitíase. Pancreatocolangiografia retrógrada endoscópica. Colecistectomia laparoscópica. Dor abdominal.

\section{INTRODUÇÃO}

A prevalência da litíase do ducto biliar comum encontra-se em torno de $10 \%$ a $15 \%$ dos pacientes com colelitíase. O seu tratamento, porém, continua controverso na literatura.

O uso de métodos endoscópicos, como a colangiopancreatografia endoscópica retrógrada (CPRE) no tratamento da coledocolitíase, é amplamente defendida por ser menos invasiva e proporcionar recuperação mais rápida ${ }^{1,3,9,13}$, apesar da exploração das vias biliares por laparoscopia apresentar o mesmo nível de resolução, com custo menor e menor taxa de complicação ${ }^{5,7,8,11,12}$.

$\mathrm{O}$ insucesso do tratamento por CPRE encontra-se na literatura em torno de $6 \%$ a $13 \%{ }^{1,8,10}$, sendo a não cateterização da papila uma das causas. A colecistectomia por videolaparoscopia com cateterização do ducto cístico, introdução de fio guia e passagem dele pela papila para direcionar a CPRE intra-operatória, torna-se importante alternativa ${ }^{1,8,9,13,14}$.

Trabalho realizado no Serviço de Cirurgia Geral I do Hospital São Rafael ${ }^{1}$ e no Departamento de Cirurgia da Escola Bahiana de Medicina e Saúde Pública ${ }^{2}$, Salvador, BA, Brasil.

Endereço para correspondência: Paulo Cezar Galvão do Amaral, e-mail: cirurgias@terra.com.br
O objetivo deste trabalho é relatar o caso de uma paciente que necessitou da realização de CPRE intraoperatória para resolução de coledocolitíase tratada sem êxito por CPRE.

\section{RELATO DO CASO}

E.M.L.D., 46 anos, sexo feminino, branca, médica, solteira, natural e procedente de Salvador, BA. Deu entrada no hospital com dor abdominal há aproximadamente 24 horas. Ela referia estar bem, quando há cerca de $24 \mathrm{~h}$ da admissão passou a evoluir com dor abdominal tipo queimação, principalmente em hipogástrio e hipocôndrio direito, irradiada para dorso, de moderada intensidade, com piora progressiva e melhora com uso de analgésico. Relata dois episódios prévios semelhante há um mês. O exame físico mostrou abdome doloroso à palpação em epigástrio e hipocôndrio direito, sem irritação peritonial. Análises clínicas realizadas (bilirrubinas totais e frações, fosfatase alcalina e GGT) não apresentaram alterações dignas de nota por estarem na faixa da normalidade. Ultrasonografia de abdome evidenciou colecistolitíase associada à coledocolitíase, com tamanho do colédoco menor que $0,8 \mathrm{~cm}$ (Figuras 1e 2). 


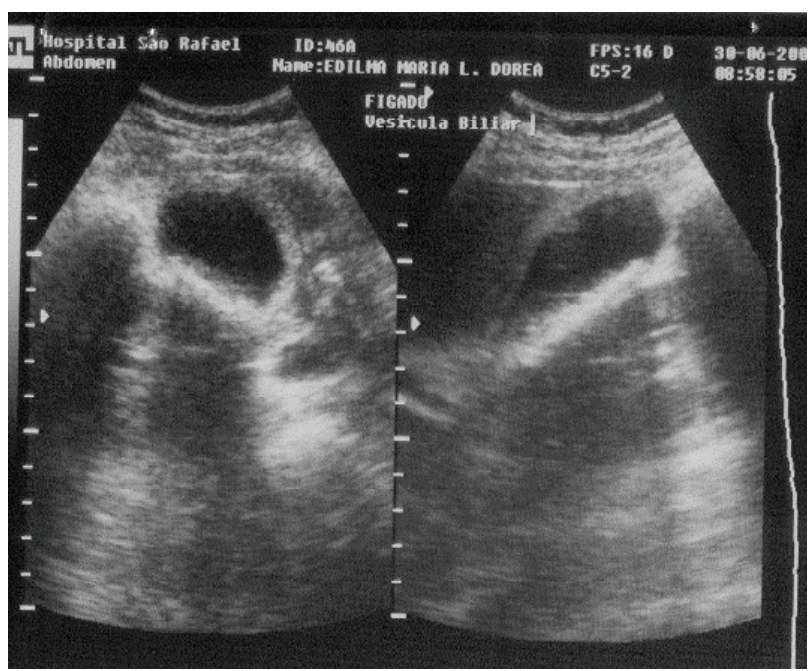

FIGURA 1 - Estudo ultra-sonográfico mostrando colecistolitíase com cálculos múltiplos de pequeno tamanho e sombra acústica bem definida

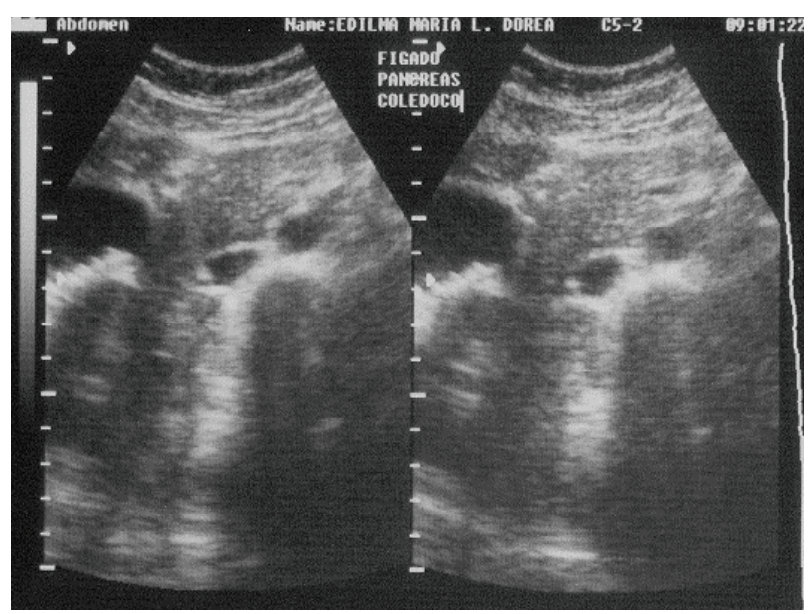

FIGURA 2 - Estudo ultra-sonográfico mostrando coledocolitíase em ducto de calibre dentro da normalidade

Indicou-se, então, CPRE e quando de sua realização não foi possível a cateterização da papila devido a ela ser pouco protusa com óstio único, cateterizando-se o ducto pancreático principal com sucesso, revelando pancreatografia normal (Figura 3). Foi então indicada CPRE intraoperatória como opção terapêutica.

Iniciada videolaparoscopia com identificação, dissecção e clipagem superior do ducto cístico, foi passado o fio guia longo da CPRE por punção percutânea com agulha $18 \mathrm{~F}$ em hipocôndrio direito até acessar a luz do ducto cístico, permitido passagem do fio guia até a papila hepatopancreática. Feita preensão do fio guia no duodeno através do duodenoscópio, que serviu para orientar a passagem do papilótomo, prosseguindo então a CPRE, com esfincterotomia e limpeza da via biliar com a retirada de três cálculos de aproximadamente $0,3 \mathrm{~cm}$ cada. Completado o procedimento cirúrgico para colecistectomia da forma habitual com extração retrógrada da vesícula biliar do leito hepático pelo portal umbilical.

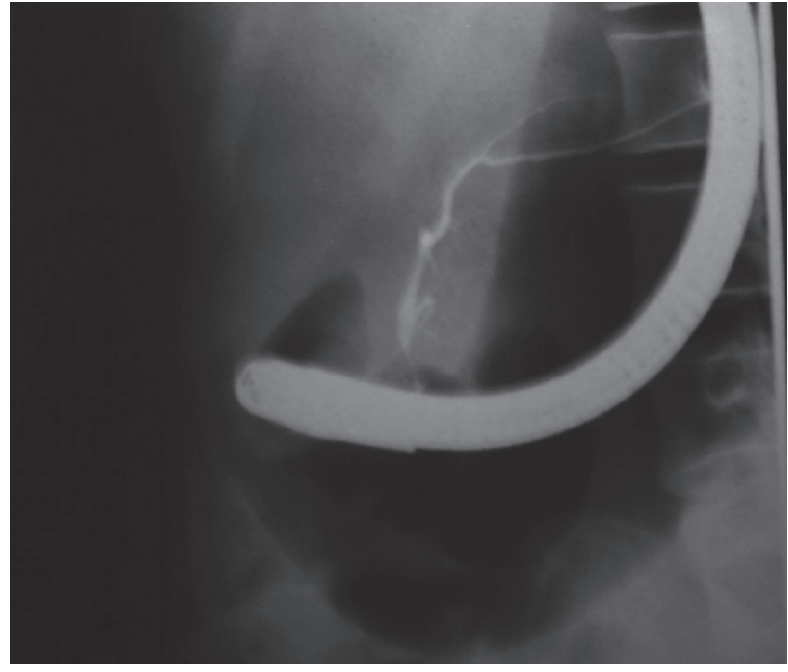

FIGURA 3 - Pancreatografia normal

A paciente evoluiu no pós-operatório imediato com queixa de leve dor abdominal e náuseas. Cursou com melhora progressiva usando sintomáticos, aceitando bem a dieta após $18 \mathrm{~h}$ do procedimento. Obteve alta hospitalar no $2^{\circ}$ dia do pós-operatório. Não se realizou dosagem de amilase no pós-operatório.

\section{DISCUSSÃO}

O tratamento da coledocolitíase após falha terapêutica da CPRE pode ser realizado por algumas alternativas, não havendo ainda consenso na literatura ${ }^{1,2,4,5,6,7,8,9,10,11,12,13,14}$.

A exploração de vias biliares unicamente por via laparoscópica é indicada por alguns autores como padrão-ouro para o tratamento da coledocolitíase $e^{5,6,7,8,9,10,11}$. A depender das condições cirúrgicas, $\mathrm{o}$ acesso à via biliar pode ser feito através do ducto cístico ou do colédoco, porém apresentando maior morbidade e maior permanência hospitalar. Relatam-se taxas de resolução da coledocolitíase de $85,7 \%$ a 97,2\% $\%^{11,12,13}$ com complicações de 2,9 a $9,1 \%{ }^{5,6,7,8,9,10,11}$.

A CPRE intra-operatória é outra opção para este tratamento. A possibilidade de cateterização do ducto cístico com a passagem de fio guia pela papila, facilita a realização do procedimento podendo-se evitar a manipulação e abertura direta da via biliar. A taxa de sucesso varia de $82,7 \%$ a $89 \%$ com complicações de $5,6 \%$ a $12,3 \%^{1,13}$.

A exploração de vias biliares pelo método convencional reserva-se atualmente a situações especiais como impossibilidade de utilizar os métodos anteriores ${ }^{1,3,11}$.

\section{CONCLUSÃO}

A abordagem da coledocolitíase por CPRE intra-operatória, guiada por cateterização da papila hepatopancreática através do ducto cístico seguida de colecistectomia videolaparoscópica mostrou-se segura e eficaz. 
Ettinger Jr E, Amaral PCG, Azaro-Filho E, Fortes MF, Cangussu HC, Fahel E. Intraoperative endoscopic retrograde cholangiopancreatography as an alternative for choledocholithiasis treatment. ABCD Arq Bras Cir Dig 2007;20(1):65-7.

ABSTRACT - Background - Choledocholithiasis treatment is still a controversial topic, being done either with endoscopic retrograde cholangiopancreatography before laparoscopic cholecystectomy or by the use of laparoscopic cholecystectomy and exploration of common bile duct. According to literature, there is an unsuccessful rate of $6 \%$ when utilizing pre-operative endoscopic retrograde cholangiopancreatography. Case report - We present a case report of a 45-year-old patient, female, who was admitted to an emergency room with abdominal pain in the epigastrius and right hypochondrium. Ultrasonography showed choledocholithiasis, and the patient was indicated to a pre-operative endoscopic retrograde cholangiopancreatography, by which the catheterization of papilla was unsuccessful. The patient was then, submitted to a laparoscopic cholecystectomy and exploration of the common bile ducts by preoperative and intraoperative endoscopic retrograde cholangiopancreatography with the catheterization of papilla being guided by a guiding thread which was introduced through the cystic duct. A duodenoscopy with the capture of the guiding thread was performed, allowing the passage of papillotome, giving sequence to the preoperative endoscopic retrograde cholangiopancreatography, by which gallstones were removed. The patient had a satisfying recovery being discharged on the second postoperative day. Conclusion - In this case, the choledocholithiasis management by intraoperative endoscopic retrograde cholangiopancreatography with laparoscopic cholecystectomy was considered both safe and effective.

HEADINGS - Choledocholithiasis. Cholangiopancreatography, endoscopic retrograde. Cholecystectomy, laparoscopic. Abdominal pain.

\section{REFERÊNCIAS}

1 Basso N, Pizzuto G, Desdemona S, Materia A, Gianfranco S, Fantini A, Fioca F, Trentino P. Laparoscopic cholecystectomy and intraoperative endoscopic sphincterotomy in the treatment of cholecysto-choledocholithiasis. Gastrointest Endosc, 50(4):532-5, 1999.

2 Dorman JP, Franklin ME, Glas JL. Laparoscopic common bile duct exploration by choledochotomy. An effective and efficient method of treatment of choledocholithiasis. Surg Endosc, 12(7):926-8, 1998.

3 Fahel E. Colelitíase. In: Fahel E, Amaral P, Ázaro E, ed. Manual de Atualização em Cirurgia Geral: Diagnóstico e Tratamento. Rio de Janeiro: Revinter; 2001. p.3-16.

4 Fernandez M, Csendes A, Yarmuch J, Diaz H, Silva J. Management of common bile duct stones: the state of the art in 2000. Int Surg, 88(3):159-63, 2003.

5 Heili MJ, Wintz NK, Fowler DL. Choledocholithiasis: endoscopic versus laparoscopic management. Am Surg, 65(2):135-8, 1999.

6 Hyser MJ, Chaudhry V, Byrne MP. Laparoscopic transcystic management of choledocholithiasis. Am Surg, 65(7):606-9, 1999.

7 Liberman MA, Phillips EH, Carroll BJ, Fallas MJ, Rosenthal R, Hiatt J. Cost-effective management of complicated choledocholithiasis: laparoscopic transcystic duct exploration or endoscopic sphincterotomy. J Am Coll Surg, 182(6):488-94, 1996.

8 Liu CL, Lai E CS, Lo CM, Chu KM, Fan ST, Wong J. Combined laparoscopic and endoscopic approach in patients with cholelithiasis and choledocholithiasis. Surgery, 119(5):534-7, 1996.
9 Moroni J, Haurie JP, Judchak I, Fuster S. Single-stage laparoscopic and endoscopic treatment for choledocholithiasis: a novel approach. J Laparoendosc Adv Surg Tech A, 9(1):69-74, 1999.

10 Pereira-Lima JC, Rynkowski CB, Rodhen EL. Endoscopic treatment of choledocholithiasis in the era of laparoscopic cholecystectomy: prospective analysis of 386 patients. Hepatogastroenetroloy, 48(41):1271-41, 2001.

11 Petelin JB. Laparoscopic common bile duct exploration. Surg Endosc, 17(11):1705-15, 2003

12 Sgourakis G, Karaliotas K. Laparoscopic common bile duct exploration and cholecystectomy versus endoscopic stone extraction and laparoscopic cholecystectomy for choledocholithiasis. A prospective randomized study. Minerva Chir, 57(4):467-74, 2002.

13 Wei Q, Wang JG, Li LB, Li JD. Management of choledocholithiasis: comparison between laparoscopic common bile duct exploration and intraoperative endoscopic sphincterotomy. World J Gastroenterol, 9(12):2856-8, 2003.

14 Wright BE, Freeman ML, Cumming JK, Quickel RR, Mandal AK. Current management of common bile duct stones: is there a role for laparoscopic cholecystectomy and intraoperative endoscopic retrograde cholangiopancreatography as a single-stage procedure?. Surgery, 132(4):729-35, 2002.

Conflito de interesse: não há Fonte financiadora: não há Recebido para publicação em: 16/08/2006 Aceito para publicação em: 05/12/2006 